\title{
Analysis of different impact rates on the forecasts cost of building project using sensitivity analysis techniques
}

\author{
Godwin Adie Akeke ${ }^{1, *}$ and Melody Sunday Osok ${ }^{2}$ \\ ${ }^{1}$ Department of Civil Engineering, University of Cross River State, Calabar. \\ ${ }^{2}$ Department of Civil Engineering, University of Nigeria, Nsukka.
}

World Journal of Advanced Engineering Technology and Sciences, 2021, 04(01), 040-051

Publication history: Received on 20 November 2021; revised on 29 December 2021; accepted on 31 December 2021

Article DOI: https://doi.org/10.30574/wjaets.2021.4.1.0083

\begin{abstract}
Over the years, Life Cycle Costing (LCC) has been recognized and used as an important technique for evaluating, forecasting and discounting the future costs of building to the present day value, from conception, design to completion, operation, maintenance, down to decommissioning. This work presents a study of Analysis on different discount rate of the forecasts cost of building project using sensitivity analysis techniques, the case study being Calabar International Conference Center (CICC) building project. Life cycle cost analysis wasconducted and forecast for 51 years using Net present value (NPV) with the following discount rates $4 \%, 5 \%, 6 \%, 8 \%, 10 \%, 12 \%$ and $13 \%$ respectively. Results showed that the lower the discount rates, the higher the cost value and via vasa. The building had a positive value $>0$ indicating a significant benefit at the end of the study period. The percentage contribution of the discount rate on the initial cost, salvage value and the life cycle cost indicates that at $4 \%$ the initial cost accounted for $85 \%$ of the discounted cost, life cycle cost $13 \%$ and salvage value $2 \%$. The salvage value recorded $0 \%$ at $12 \%$ and $13 \%$ discount rate The higher the discount rates the higher the discounted initial cost and the lower the life cycle cost.
\end{abstract}

Keywords: Life cycle cost; Discount rates; Sustainability assessment; Forecasting; Building industry, Sensitivity analysis

\section{Introduction}

The concept of building life cycle costing (LCC) was first applied in the procurement of military equipment by the United StateDepartment of Defense in the mid-1960s. [1] Since then, researchers and academia had further developed LCC frameworks used in many sectors including the building industry. The LCC concept is also used in evaluating the total cost of project ownership and how best it can minimize project cost for good return of investment. The project ownership cost consist of the design and construction, operation, maintenance, replacement and decommissioning of the building, and there are the most costs oriented stages to be considered during life cycle analysis. Furthermore, LCC can be used to evaluate future cost of operation, maintenance and replacement of buildings. Sensitivity analyses of different discount rates are used in life cycle analysis to discount the future cost of a building project to its present value. This paper, attempt to using different discount rate to evaluate the future cost of CICC building project for a forecast period of 51 years.

\subsection{Basic Steps of Life Cycle Cost}

There have been numerous research works on possible steps to generate an efficient LCC analysis such as [2]; [3]; [4]; [5], as quoted by [6]. However, in every set of steps the same essential points can be identified. The basic LCC steps used in making decisions about options are as follows;

\footnotetext{
* Corresponding author: Godwin Adie Akeke

Department of Civil Engineering, University of Cross River State, Calabar.

Copyright ( 12021 Author(s) retain the copyright of this article. This article is published under the terms of the Creative Commons Attribution Liscense 4.0.
} 
- Identify project objectives, options and constraints and developing a framework

- $\quad$ Establish basic assumptions with respect to the period of study and the discount rate

- $\quad$ Compile data;

- $\quad$ Discount cash flows to a comparable time base, then carry out analysis, iteration required;

- $\quad$ Compute total life cycle costs, compare options and make decisions.

\subsection{Life cycle cost of building project}

[7] work on life cycle cost calculation models for buildings highlighted six different methods for economic evaluation of life cycle costing. The methods identify what it could calculate,itadvantages and disadvantage and when it should be used. The study also highlights different typesof data for life cycle costing calculation, such as occupancy data, physical data, performance data and quality data. The approach identify net present value (NPV) methodology as one of the most suitable tool for life cycle costs analysis, but in cases were alternative scheme with different life cycle has to be compared, the equivalent annual cost (ECA) is the best alternative. The concept oflife cycle costing technique is used in evaluating the net cost of building component and the cost comparison of different materials throughout their service life. The concept gives a guide on its analysis to forecast its parameters, explain its results, bring out the limitations associated in its practice that highlight the disadvantages and uncertainty in the economic evaluation [8]. A framework from [9] suggest that building frames, upper floor, external/internal walls and partitions, roofs, doors, windows, floor finishes and decorations and building services are the most important component for which LCC analysis can be applied. Life- cycle cost (LCC) of a building is the total cost of owning, operating, and maintaining a planned project over its useful life from cradle to grave. That is, it includes not only the acquisition and energy costs, but also the maintenance cost and the salvage value for a better performance [10].

The life cycle cost analysis method can be used to determine whether proceeding with energy conservation is cost effective, or to compare the economic consequences of alternative design solutions. [1] approach attempt to examine the practical usefulness of LCC approach and the theoretical perceptions in achieving environmentally responsible investment.

Theapproach outlines the following limitations of LCC: inability to absorb common decision, negatingfuture generation costs, unreliable data, complexity and failure to take into account the decision- maker unprofessional ability to handle risk. Findings from [5] outline the limitations surrounding the used of LCC data analysis such as difficulties in downloading data, non-accurate data, missing data, high data variation and limited data. [11] believed that the neoclassical economic theory has fail to handle decision-making uncertainty, available findings anduse of discounting techniques in estimating future costs. Hence, unreliable, non-availability and poor quality of data are assumed to be the major limitation of LCC analysis. Developing an LCC data base was suggested by [12] to eradicate the data challenges such as Open system for construction (OSCON) data base system created to enhance information integration. Hence, the data base for this research work will be gotten from Calabar International Conference Centre (CICC) project, the central bank of Nigeria, the project quantity surveyor and order relevant sources.

Life cycle costs generally consist of the following. Acquisition cost: It is the purchasing cost or first cost and includes taxes, fees, delivery,installation an initial start-up. Annual owning and operating costs: Include those for maintenance, taxes, insurance, interest on borrowed money, and any other recurring costs over the useful life. Some ofthose costs may vary with age and inflation. Terminal value: It is the disposal cost or deduction form costs if a salvage value, computed at the end of the useful life of a project [13].

\subsection{Present worth method}

This process incorporates the cost benefit that is acquired during the project life cycle. However, the net present value (NPV) is the difference between the cost benefits against the assessments. The cost benefit analysis is used to weigh all expected cost base on discounting principles. This method is called NPV method. This method is carried out by reducing each cash flow and out flow at certain interval, to its closest present value, [14].

The mathematical relation is:

$$
N P V=\sum_{t=0}^{N} \frac{\mathrm{C}_{t}}{(1+r)^{t}}
$$


$\mathrm{t}=$ time of the cash flow

$\mathrm{N}=$ the total time of the cash inflow/ out flow in the project

$\mathrm{R}=$ the discount rate (the rate of return that could be earned on an investment in the financialmarket with similar risk)

$\mathrm{C}_{\mathrm{t}}=$ the net cash flow (the amount of cash) at time, $\mathrm{t}$

\section{Methodology and sampling technique}

The net present value (NPV) technique was used to analysis all cash inflow and out flow of the project. The parameters for the study were gotten from the CICC project, they includethe Initial cost (Construction cost), Operation cost, Maintenance/Repair cost and Replacement cost, why the salvage value and the life cycle cost is determined at the end of the study period. The Initial cost of the project consist of the design cost and construction cost. Since CICC project is a government project the cost of land and taxation is not included. The Operation cost (PC) of the project includethe cost of water bills, electricity bills (independent power source and National power source) cleaning and garbage disposal wages of staffs and other costs. The Maintenance and Repair cost (M/RC) consist of the maintenance and repair of doors, windows, plaster of Paris (POP), roofs, electrical fittings, plumbing, fire protection system, fumigation and other costs. Replacement cost (RC) include the cost of doors and windows, appliances, chairs and tables, electrical services, plumbing and other costs.

This costs drivers are collected for each month and sum up to obtain the yearly costs of the facility for every year. The sum of $\mathrm{PC}+\mathrm{M} / \mathrm{RC}+\mathrm{RC}$ represent thesustaining cost of CICC project. An estimated discount rate $\mathrm{r}$ was adopted for the project life cycle costs (LCC), where it was discounted to a forecasted point time.

\subsection{Discounted cash flow techniques}

The equation for discounted cash flow is obtained by integrating future cash into respective NPV. The discounted cash flow relation is given as follows:

$$
\mathrm{DPV}=\mathrm{FV}=\mathrm{FV}(1-\mathrm{d}) \mathrm{t}=\mathrm{FV} \llbracket(1+\mathrm{i})-\rrbracket \wedge \mathrm{t}(1+\mathrm{i})^{\wedge} \mathrm{t}(2)
$$

Where

$\mathrm{DPV}=$ the discounted present value of the future cash flow $(\mathrm{FV})$ at the time point $\mathrm{t}$;

$\mathrm{FV}=$ the nominal value of the cash flow amount in a future period; FV can be substituted with Ct

$\mathrm{i}=$ the interest rate, the interest rate shows the cost of putting down the capital and not carrying out any other investment. It could also give room for payment related risks

$\mathrm{d}=$ the discount rate, $\mathrm{i} /(1+\mathrm{i})$, i.e., the interest rate presented as a substitute at the beginning in place of adding at the end of the said year;

$t=$ the number of years' entail for the future cash flow to take place In many occasions, there are usually many future cash flow lingering in project assessment. The following relation is use to express discounted cash flow in different time:

$$
N P V=\sum_{t=0}^{N} \frac{F V_{t}}{(1+r)^{t}}=\sum_{t=0}^{N} \frac{C_{t}}{(1+r)^{t}} \text { (3) }
$$

Equation (3) above represents the future cash flow (FV) at time (t), which demonstrate the effect of the interest rate on products and services throughout the period of study.

\section{Results and discussion}

This section presents detail results on the sustainability assessment of building life cycle cost and the development of LCC model of CICC project Calabar including the discussion of findings.

\subsection{Inflation rate}


This represent consumer price index (CPI) for various price of product and services. The consumer price index (CPC) shows the percentage change of price of products and services of various commodities World bank (2019). The analysis was carried out from 2000 to 2018 to show the rate of inflation on market commodities.

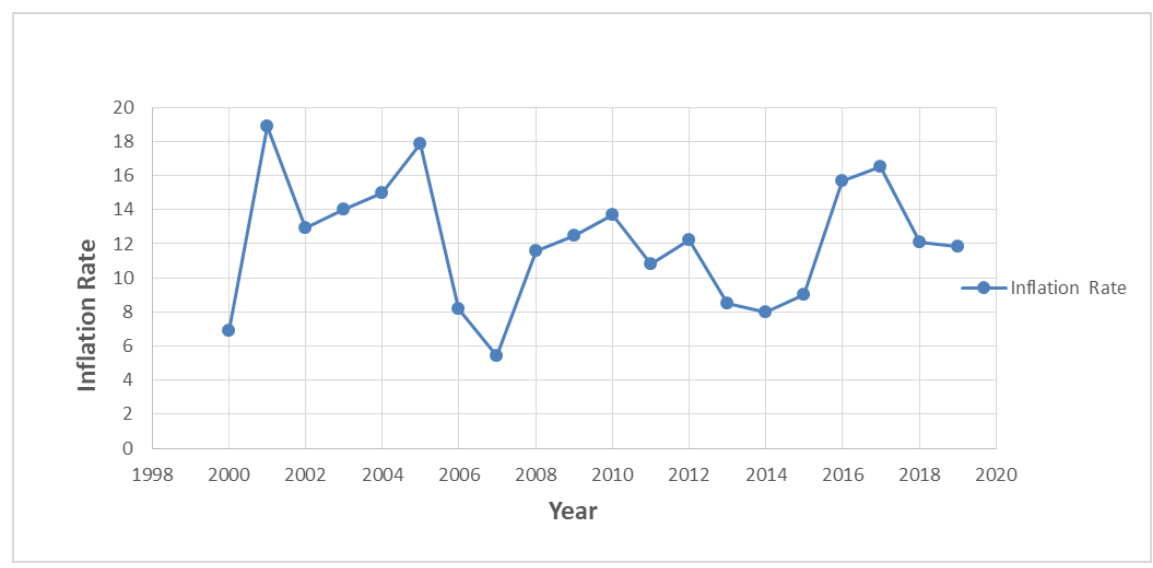

Figure 1 Inflation rate

\subsection{Total forecast cost of the case study}

Total forecast cost showing the cost of operation, maintenance and replacement of the project for the period of 51 years under review.

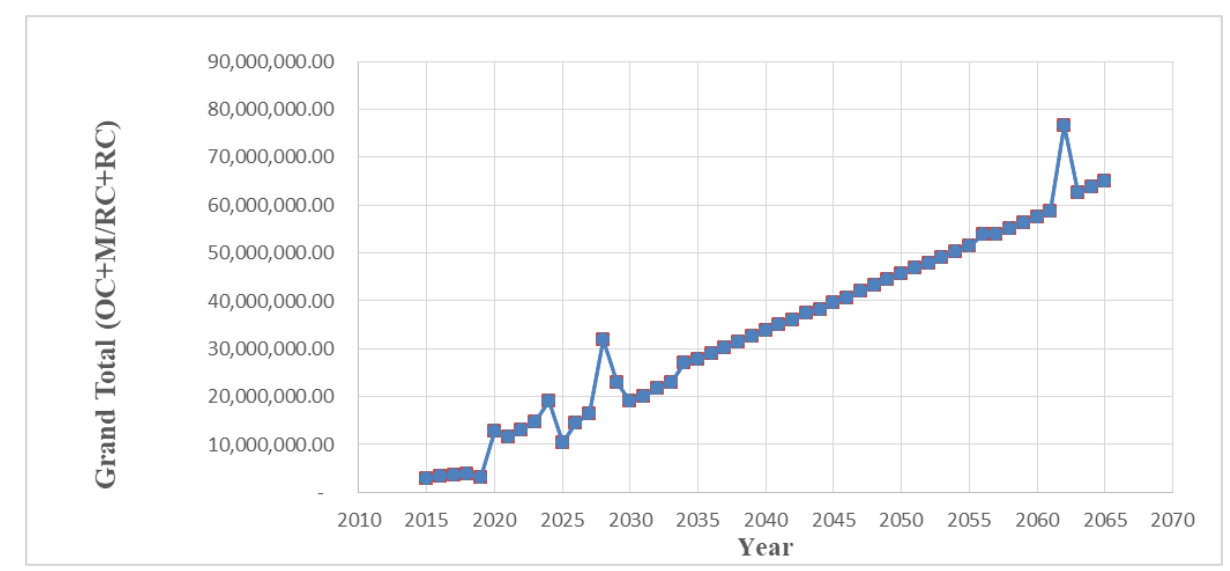

Figure 2 Total forecast cost

\subsection{Sensitivity analysis of discount rate}

The discount rates adopted for this study are $4 \%, 4.5 \%, 5 \%, 6 \%, 8 \%, 12 \%$ and $13 \%$ respectively.The values were used to discount all future cost to the base year including the annual discount factor which represents the cumulative percentage rate for the discounted values used for economicanalysis when determining the equivalent value of different alternative. The discount process involves the following steps: identifying the requite discount rate, sum total of operational, Maintenance/Repair and Replacement costs, the number of years, the discount factor and the discount rate of the cash flow. While the salvage value of CICC building complex was computed by a professional estate surveyor Egbe and partners Nigeria Ltd. The life cycle cost of the case study was obtained using the adopted discount rates to determine the sensitivity of the analysis for the study. It was observed when discounting the future cost to the present values; the highest discount rate at $13 \%$ generated the lowest cost values while the list discount of $4 \%$ generated the highest cost value. 
Table 1 Discount rate of $4 \%$

\begin{tabular}{|c|c|c|c|c|c|c|c|c|c|}
\hline YEAR & $\mathbf{T}$ & $F=(O C+M / R C+R C)$ & $1=(1+r)^{\wedge} t$ & $F=(1+r)^{\wedge} t$ & Year & $\mathbf{t}$ & $\mathrm{F}(\mathrm{OC}+\mathrm{M} / \mathrm{RC}+\mathrm{RC})$ & $1=(1+r)^{\wedge} t$ & $F(1+r)^{\wedge} t$ \\
\hline 2015 & 0 & 2865400 & 1 & & & & & & \\
\hline 2016 & 1 & 3348600 & 0.961538 & 3219807.7 & 2041 & 26 & 34944275.02 & 0.360689 & 12604024 \\
\hline 2017 & 2 & 3640000 & 0.924556 & 3365384.6 & 2042 & 27 & 36062822.09 & 0.346817 & 12507184 \\
\hline 2018 & 3 & 3847060 & 0.961538 & 3420022.3 & 2043 & 28 & 37313975.68 & 0.333477 & 12443370 \\
\hline 2019 & 4 & 3026000 & 0.854804 & 2586637.5 & 2044 & 29 & 38257237.63 & 0.961538 & 11964779 \\
\hline 2020 & 5 & 12720050 & 0.821927 & 10454954 & 2045 & 30 & 39660000.16 & 0.308319 & 11795421 \\
\hline 2021 & 6 & 11651476 & 0.790315 & 9208330.7 & 2046 & 31 & 40560029.59 & 0.29646 & 11757614 \\
\hline 2022 & 7 & 13066616.29 & 0.759918 & 9929554.5 & 2047 & 32 & 41999428.07 & 0.285058 & 11561958 \\
\hline 2023 & 8 & 14673756.57 & 0.73069 & 10721970 & 2048 & 33 & 43175672.26 & 0.274094 & 11511799 \\
\hline 2024 & 9 & 18993341.3 & 0.702587 & 13344470 & 2049 & 34 & 44370600.89 & 0.263552 & 11693965 \\
\hline 2025 & 10 & 10389272.06 & 0.675564 & 7018619.9 & 2050 & 35 & 45556721.14 & 0.253415 & 11244197 \\
\hline 2026 & 11 & 14517669.52 & 0.649581 & 9430401.3 & 2051 & 36 & 46742841.39 & 0.243669 & 11100748 \\
\hline 2027 & 12 & 16355050.88 & 0.624597 & 10215317 & 2052 & 37 & 47921203.62 & 0.234297 & 10951700 \\
\hline 2028 & 13 & 31882417.3 & 0.600574 & 19147754 & 2053 & 38 & 49114265.25 & 0.225285 & 10795949 \\
\hline 2029 & 14 & 22927432.07 & 0.577475 & 13240021 & 2054 & 39 & 50300354.09 & 0.216621 & 10639162 \\
\hline 2030 & 15 & 18972446.83 & 0.555265 & 10534726 & 2055 & 40 & 51486442.93 & 0.208289 & 10477013 \\
\hline 2031 & 16 & 20017461.6 & 0.533908 & 10687486 & 2056 & 41 & 53765790.75 & 0.200278 & 10311598 \\
\hline 2032 & 17 & 21612476.37 & 0.513373 & 11095267 & 2057 & 42 & 53962740.52 & 0.192575 & 10353943 \\
\hline 2033 & 18 & 22851568.52 & 0.493628 & 11280177 & 2058 & 43 & 55152461.45 & 0.185168 & 9992183.7 \\
\hline 2034 & 19 & 26925820.44 & 0.474642 & 12780137 & 2059 & 44 & 56342182.38 & 0.178046 & 9819694.4 \\
\hline 2035 & 20 & 27804571.51 & 0.456387 & 12689643 & 2060 & 45 & 57531903.31 & 0.171198 & 9645692.1 \\
\hline 2036 & 21 & 28994522.1 & 0.438834 & 12723771 & 2061 & 46 & 58721624.24 & 0.164614 & 9470548.5 \\
\hline 2037 & 22 & 30184472.68 & 0.421955 & 12736501 & 2062 & 47 & 76623022.82 & 0.158283 & 9294608.7 \\
\hline 2038 & 23 & 31374423.26 & 0.405726 & 12729430 & 2063 & 48 & 62493705.9 & 0.152195 & 11661623 \\
\hline 2039 & 24 & 32564373.85 & 0.390121 & 12704062 & 2064 & 49 & 63726058.66 & 0.146341 & 9145398.9 \\
\hline 2040 & 25 & 33754324.43 & 0.375117 & 12661814 & 2065 & 50 & 64958411.42 & 0.140713 & 9140468 \\
\hline
\end{tabular}

Table 2 Discount rate of $4.5 \%$

\begin{tabular}{|c|c|c|c|c|c|c|c|c|c|}
\hline YEAR & $\mathbf{t}$ & $F=(O C+M / R C+R C)$ & $1(1+r)^{\wedge} t$ & $F=(1+r)^{\wedge} t$ & Year & $\mathbf{t}$ & $F=(O C+M / R C+R C)$ & $1(1+r)^{\wedge} t$ & $F=(1+r)^{\wedge} t$ \\
\hline 2015 & 0 & 2861400 & 1 & & & & & & \\
\hline 2016 & 1 & 3102000 & 0.9569378 & 2968421.05 & 2041 & 26 & 33992127.25 & 0.3184025 & 10823177.78 \\
\hline 2017 & 2 & 3388400 & 0.91572995 & 3102859.37 & 2042 & 27 & 35082512.77 & 0.3046914 & 10689338.99 \\
\hline 2018 & 3 & 3657860 & 0.8762966 & 3205370.3 & 2043 & 28 & 36305504.81 & 0.2915707 & 10585621.16 \\
\hline 2019 & 4 & 24818000 & 0.83856134 & 20811415.4 & 2044 & 29 & 37207543.66 & 0.279015 & 10381463.4 \\
\hline 2020 & 5 & 11811450 & 0.80245105 & 9478110.41 & 2045 & 30 & 38593464.63 & 0.267 & 10304455.65 \\
\hline
\end{tabular}




\begin{tabular}{|l|l|l|l|l|l|l|l|l|l|}
\hline 2021 & 6 & 10941156 & 0.76789574 & 8401667.06 & 2046 & 31 & 39464760.81 & 0.2555024 & 10083341.39 \\
\hline 2022 & 7 & 12681433.43 & 0.73482846 & 9318678.17 & 2047 & 32 & 40876339.97 & 0.2444999 & 9994261.492 \\
\hline 2023 & 8 & 13969639.43 & 0.70318513 & 9823242.68 & 2048 & 33 & 42024335.56 & 0.2339712 & 9832484.51 \\
\hline 2024 & 9 & 19633845.43 & 0.67290443 & 13211701.5 & 2049 & 34 & 43191015.6 & 0.2238959 & 9670290.952 \\
\hline 2025 & 10 & 11109375.24 & 0.64392768 & 7153634.25 & 2050 & 35 & 44348887.25 & 0.2142544 & 9501946.085 \\
\hline 2026 & 11 & 12519669.52 & 0.61619874 & 7714604.57 & 2051 & 36 & 45506758.91 & 0.2050282 & 9330167.685 \\
\hline 2027 & 12 & 15640118.08 & 0.58966386 & 9222412.47 & 2052 & 37 & 46656956.81 & 0.1961992 & 9154058.049 \\
\hline 2028 & 13 & 31117556.53 & 0.56427164 & 17558754.7 & 2053 & 38 & 47821694.45 & 0.1877504 & 8978544.166 \\
\hline 2029 & 14 & 22112643.34 & 0.53997286 & 11940227.3 & 2054 & 39 & 48979535.04 & 0.1796655 & 8799932.292 \\
\hline 2030 & 15 & 18807730.15 & 0.51672044 & 9718338.64 & 2055 & 40 & 50137375.63 & 0.1719287 & 8620053.868 \\
\hline 2031 & 16 & 19852816.95 & 0.49446932 & 9816608.95 & 2056 & 41 & 52406521.35 & 0.1645251 & 8622186.74 \\
\hline 2032 & 17 & 21447903.76 & 0.47317639 & 10148641.6 & 2057 & 42 & 52558895.39 & 0.1574403 & 8274886.211 \\
\hline 2033 & 18 & 22678575.65 & 0.45280037 & 10268867.4 & 2058 & 43 & 53720428.02 & 0.1506605 & 8093548.528 \\
\hline 2034 & 19 & 25859604.06 & 0.43330179 & 11205012.7 & 2059 & 44 & 54881960.65 & 0.1441728 & 7912483.883 \\
\hline 2035 & 20 & 27021393.09 & 0.41464286 & 11204227.7 & 2060 & 45 & 56043493.28 & 0.1379644 & 7732005.025 \\
\hline 2036 & 21 & 28183182.11 & 0.39678743 & 11182732.3 & 2061 & 46 & 57205025.91 & 0.1320233 & 7552397.262 \\
\hline 2037 & 22 & 29344971.14 & 0.37970089 & 11142311.5 & 2062 & 47 & 73661644.31 & 0.1263381 & 9306272.351 \\
\hline 2038 & 23 & 30506760.17 & 0.36335013 & 11084635.3 & 2063 & 48 & 60802681.66 & 0.1208977 & 7350904.701 \\
\hline 2039 & 24 & 31668549.2 & 0.34770347 & 11011264.6 & 2064 & 49 & 62003232.36 & 0.1156916 & 7173252.178 \\
\hline 2040 & 25 & 32830338.22 & 0.3327306 & 10923658 & 2065 & 50 & 70599151.62 & 0.1107096 & 7816007.364 \\
\hline & \multicolumn{3}{|c|}{ Salvage Value $=145707728.80$, Initial Cost =5719455950.50, LCC $=676986105.50$} & \\
\hline
\end{tabular}

Table 3 Discount rate of 5\%

\begin{tabular}{|l|l|l|l|l|l|l|l|l|l|}
\hline YEAR & $\mathbf{T}$ & $\mathbf{F = ( O C + M / R C + R C )}$ & $\mathbf{1}(\mathbf{1 + r})^{\wedge} \mathbf{t}$ & $\mathbf{F = ( 1 + r ) \wedge}$ & $\mathbf{Y e a r}$ & $\mathbf{t}$ & $\mathbf{F}=(\mathbf{M C}+\mathbf{M} / \mathbf{R C}+\mathbf{R C})$ & $\mathbf{1}(\mathbf{I + r})^{\wedge} \mathbf{t}$ & $\mathbf{F}(\mathbf{1 + r})^{\wedge} \mathbf{t}$ \\
\hline 2015 & 0 & 2865400 & 1 & & & & & & \\
\hline 2016 & 1 & 3348600 & 0.95238095 & 3189142.86 & 2041 & 26 & 34944275.02 & 0.2812407 & 9827753.588 \\
\hline 2017 & 2 & 3640000 & 0.90702948 & 3301587.3 & 2042 & 27 & 36062822.09 & 0.2678483 & 9659366.276 \\
\hline 2018 & 3 & 3847060 & 0.8638376 & 3323235.07 & 2043 & 28 & 37313975.68 & 0.2550936 & 9518557.773 \\
\hline 2019 & 4 & 3026000 & 0.82270247 & 2489497.69 & 2044 & 29 & 38257237.63 & 0.2429463 & 9294455.137 \\
\hline 2020 & 5 & 12720050 & 0.78352617 & 9966492.01 & 2045 & 30 & 39660000.16 & 0.2313774 & 9176429.65 \\
\hline 2021 & 6 & 11651476 & 0.7462154 & 8694510.78 & 2046 & 31 & 40560029.59 & 0.2203595 & 8937786.822 \\
\hline 2022 & 7 & 13066616.29 & 0.71068133 & 9286200.24 & 2047 & 32 & 41999428.07 & 0.2098662 & 8814258.968 \\
\hline 2023 & 8 & 14673756.57 & 0.67683936 & 9931776.04 & 2048 & 33 & 43175672.26 & 0.1998725 & 8629631.264 \\
\hline 2024 & 9 & 18993341.3 & 0.64460892 & 12243277.2 & 2049 & 34 & 44370600.89 & 0.1903548 & 8446156.841 \\
\hline 2025 & 10 & 10389272.06 & 0.61391325 & 6378111.81 & 2050 & 35 & 45556721.14 & 0.1812903 & 8258990.975 \\
\hline 2026 & 11 & 14517669.52 & 0.58467929 & 8488180.7 & 2051 & 36 & 46742841.39 & 0.1726574 & 8070498.146 \\
\hline 2027 & 12 & 16355050.88 & 0.55683742 & 9107104.31 & 2052 & 37 & 47921203.62 & 0.1644356 & 7879953.45 \\
\hline 2028 & 13 & 31882417.3 & 0.53032135 & 16907926.6 & 2053 & 38 & 49114265.25 & 0.1566054 & 7691557.424 \\
\hline 2029 & 14 & 22927432.07 & 0.50506795 & 11579911.2 & 2054 & 39 & 50300354.09 & 0.149148 & 7502195.523 \\
\hline 2030 & 15 & 18972446.83 & 0.4810171 & 9126071.32 & 2055 & 40 & 51486442.93 & 0.1420457 & 7313426.916 \\
\hline
\end{tabular}


World Journal of Advanced Engineering Technology and Sciences, 2021, 04(01), 040-051

\begin{tabular}{|l|l|l|l|l|l|l|l|l|l|}
\hline 2031 & 16 & 20017461.6 & 0.45811152 & 9170229.8 & 2056 & 41 & 53765790.75 & 0.1352816 & 7273522.316 \\
\hline 2032 & 17 & 21612476.37 & 0.43629669 & 9429451.85 & 2057 & 42 & 53962740.52 & 0.1288396 & 6952539.044 \\
\hline 2033 & 18 & 22851568.52 & 0.41552065 & 9495298.72 & 2058 & 43 & 55152461.45 & 0.1227044 & 6767449.75 \\
\hline 2034 & 19 & 26925820.44 & 0.39573396 & 10655461.5 & 2059 & 44 & 56342182.38 & 0.1168613 & 6584222.613 \\
\hline 2035 & 20 & 27804571.51 & 0.37688948 & 10479250.6 & 2060 & 45 & 57531903.31 & 0.1112965 & 6403099.989 \\
\hline 2036 & 21 & 28994522.1 & 0.35894236 & 10407362.3 & 2061 & 46 & 58721624.24 & 0.1059967 & 6224296.929 \\
\hline 2037 & 22 & 30184472.68 & 0.34184987 & 10318558.1 & 2062 & 47 & 76623022.82 & 0.1009492 & 7735033.961 \\
\hline 2038 & 23 & 31374423.26 & 0.32557131 & 10214612 & 2063 & 48 & 62493705.9 & 0.0961421 & 6008276.684 \\
\hline 2039 & 24 & 32564373.85 & 0.31006791 & 10097167.3 & 2064 & 49 & 63726058.66 & 0.0915639 & 5835007.312 \\
\hline 2040 & 25 & 33754324.43 & 0.29530277 & 9967745.56 & 2065 & 50 & 64958411.42 & 0.0872037 & 5664615.574 \\
\hline \multicolumn{7}{|c|}{ Salvage Value $=145707728.80$, Initial Cost = 5719455950.50 LCC = 502311378.20 } \\
\hline
\end{tabular}

Table 4 Discount rate of $6 \%$

\begin{tabular}{|c|c|c|c|c|c|c|c|c|c|}
\hline YEAR & $\mathbf{T}$ & $F=(O C+M / R C+R C$ & $1(1+r)^{\wedge} t$ & $F(1+r)^{\wedge} t$ & YEAR & $\mathbf{T}$ & $F=(O C+M / R C+R C$ & $1(1+r)^{\wedge} t$ & $F(1+r)^{\wedge} t$ \\
\hline 2015 & 0 & 2865400 & 1 & & & & & & \\
\hline 2016 & 1 & 3348600 & .94339623 & 3159056.6 & 2041 & 26 & 34944275.02 & 0.21981 & 7681102.097 \\
\hline 2017 & 2 & 3640000 & 0.88999644 & 3239587.04 & 2042 & 27 & 36062822.09 & 0.207368 & 7478273.549 \\
\hline 2018 & 3 & 3847060 & 0.83961928 & 3230065.76 & 2043 & 28 & 37313975.68 & 0.1956301 & 7299738.402 \\
\hline 2019 & 4 & 3026000 & 0.79209366 & 2396875.42 & 2044 & 29 & 38257237.63 & 0.1845567 & 7060631.011 \\
\hline 2020 & 5 & 12720050 & 0.74725817 & 9505161.32 & 2045 & 30 & 39660000.16 & 0.1741101 & 6905207.819 \\
\hline 2021 & 6 & 11651476 & 0.70496054 & 8213830.82 & 2046 & 31 & 40560029.59 & 0.1642548 & 6662181.19 \\
\hline 2022 & 7 & 13066616.29 & 0.66505711 & 8690046.11 & 2047 & 32 & 41999428.07 & 0.1549574 & 6508122.036 \\
\hline 2023 & 8 & 14673756.57 & 0.62741237 & 9206496.41 & 2048 & 33 & 43175672.26 & 0.1461862 & 6311688.466 \\
\hline 2024 & 9 & 18993341.3 & 0.59189846 & 11242129.5 & 2049 & 34 & 44370600.89 & 0.1379115 & 6119217.518 \\
\hline 2025 & 10 & 10389272.06 & 0.55839478 & 5801315.26 & 2050 & 35 & 45556721.14 & 0.1301052 & 5927167.149 \\
\hline 2026 & 11 & 14517669.52 & 0.52678753 & 7647727.2 & 2051 & 36 & 46742841.39 & 0.1227408 & 5737252.437 \\
\hline 2027 & 12 & 16355050.88 & 0.49696936 & 8127959.23 & 2052 & 37 & 47921203.62 & 0.1157932 & 5548948.61 \\
\hline 2028 & 13 & 31882417.3 & 0.46883902 & 14947721.4 & 2053 & 38 & 49114265.25 & 0.1092389 & 5365185.86 \\
\hline 2029 & 14 & 22927432.07 & 0.44230096 & 10140825.3 & 2054 & 39 & 50300354.09 & 0.1030555 & 5183729.096 \\
\hline 2030 & 15 & 18972446.83 & 0.41726506 & 7916539.18 & 2055 & 40 & 51486442.93 & 0.0972222 & 5005624.619 \\
\hline 2031 & 16 & 20017461.6 & 0.39364628 & 7879799.37 & 2056 & 41 & 53765790.75 & 0.091719 & 4931346.982 \\
\hline 2032 & 17 & 21612476.37 & 0.37136442 & 8026104.72 & 2057 & 42 & 53962740.52 & 0.0865274 & 4669255.685 \\
\hline 2033 & 18 & 22851568.52 & 0.35034379 & 8005905.15 & 2058 & 43 & 55152461.45 & 0.0816296 & 4502074.665 \\
\hline 2034 & 19 & 26925820.44 & 0.33051301 & 8899333.98 & 2059 & 44 & 56342182.38 & 0.0770091 & 4338859.563 \\
\hline 2035 & 20 & 27804571.51 & 0.31180473 & 8669596.83 & 2060 & 45 & 57531903.31 & 3654324.5 & 4179697.053 \\
\hline 2036 & 21 & 28994522.1 & 0.2941554 & 8528895.32 & 2061 & 46 & 58721624.24 & 0.0685378 & 4024651.289 \\
\hline 2037 & 22 & 30184472.68 & 0.2775051 & 8376345.02 & 2062 & 47 & 76623022.82 & 0.0646583 & 4954314.974 \\
\hline
\end{tabular}


World Journal of Advanced Engineering Technology and Sciences, 2021, 04(01), 040-051

\begin{tabular}{|c|c|c|c|c|c|c|c|c|c|}
\hline 2038 & 23 & 31374423.26 & 0.26179726 & 29598512.5 & 2063 & 48 & 62493705.9 & 0.0609984 & 3812016.279 \\
\hline 2039 & 24 & 32564373.85 & 0.24697855 & 8042701.78 & 2064 & 49 & 63726058.66 & 0.0575457 & 3667158.33 \\
\hline 2040 & 25 & 33754324.43 & 0.23299863 & 7864711.37 & 2065 & 50 & 64958411.42 & 0.0542884 & 3526485.742 \\
\hline \multicolumn{8}{|c|}{ Salvage Value $=145707728.80$, Initial Cost $=5719455950.50$ LCC $=257714200.10$} \\
\hline
\end{tabular}

Table 5 Discount rate of $8 \%$

\begin{tabular}{|c|c|c|c|c|c|c|c|c|c|}
\hline YEAR & $\mathbf{t}$ & $F=(O C+M / R C+R C$ & $1(1+r)^{\wedge} t$ & $F(1+r)^{\wedge} t$ & Year & $\mathbf{t}$ & $F=(O C+M / R C+R C)$ & 1.08 & $F(1+r)^{\wedge} t$ \\
\hline 2015 & 0 & 2865400 & 1 & & & & & $1(1+r)^{\wedge} t$ & \\
\hline 2016 & 1 & 3348600 & 0.92592593 & 3100555.56 & 2041 & 26 & 34944275.02 & 0.1352018 & 4724527.617 \\
\hline 2017 & 2 & 3640000 & 0.85733882 & 3120713.31 & 2042 & 27 & 36062822.09 & 0.1251868 & 4514589.958 \\
\hline 2018 & 3 & 3847060 & 0.79383224 & 3053920.26 & 2043 & 28 & 37313975.68 & 0.1159137 & 4325201.755 \\
\hline 2019 & 4 & 3026000 & 0.73502985 & 2224200.33 & 2044 & 29 & 38257237.63 & 0.1073275 & 4106054.404 \\
\hline 2020 & 5 & 12720050 & 0.6805832 & 8657052.3 & 2045 & 30 & 39660000.16 & 0.0993773 & 3941305.025 \\
\hline 2021 & 6 & 11651476 & 0.63016963 & 7342406.28 & 2046 & 31 & 40560029.59 & 0.092016 & 3732173.656 \\
\hline 2022 & 7 & 13066616.29 & 0.5834904 & 7624245.1 & 2047 & 32 & 41999428.07 & 0.0852 & 3578353.164 \\
\hline 2023 & 8 & 14673756.57 & 0.54026888 & 7927774.09 & 2048 & 33 & 43175672.26 & 0.0788889 & 3406082.613 \\
\hline 2024 & 9 & 18993341.3 & 0.50024897 & 9501399.37 & 2049 & 34 & & 0.0730453 & 3241064.125 \\
\hline 2025 & 10 & 10389272.06 & 0.46319349 & 4812243.17 & 2050 & 35 & 45556721.14 & 0.0676345 & 3081208.001 \\
\hline 2026 & 11 & 14517669.52 & 0.42888286 & 6226379.62 & 2051 & 36 & 46742841.39 & 0.0626246 & 2927250.65 \\
\hline 2027 & 12 & 16355050.88 & 0.39711376 & 6494815.73 & 2052 & 37 & 47921203.62 & 0.0579857 & 2778745.45 \\
\hline 2028 & 13 & 31882417.3 & 0.36769792 & 11723098.7 & 2053 & 38 & 49114265.25 & 0.0536905 & 2636968.506 \\
\hline 2029 & 14 & 22927432.07 & 0.34046104 & 7805897.4 & 2054 & 39 & 50300354.09 & 0.0497134 & 2500602.024 \\
\hline 2030 & 15 & 18972446.83 & & 5980906.49 & 2055 & 40 & & 0.0460309 & 2369969.021 \\
\hline 2031 & 16 & 20017461.6 & 0.29189047 & 5842906.23 & 2056 & 41 & 53765790.75 & 0.0426212 & 2291564.378 \\
\hline 2032 & 17 & 21612476.37 & 0.27026895 & 5841181.33 & 2057 & 42 & 53962740.52 & 0.0394641 & 2129591.315 \\
\hline 2033 & 18 & 22851568.52 & 0.25024903 & 5718582.84 & 2058 & 43 & 55152461.45 & 0.0365408 & 2015317.211 \\
\hline 2034 & 19 & 26925820.44 & 0.23171206 & 6239037.43 & 2059 & 44 & 56342182.38 & 0.0338341 & 1906287.603 \\
\hline 2035 & 20 & 27804571.51 & 0.21454821 & 5965420.98 & 2060 & 45 & 57531903.31 & 1575803.4 & 1802352.548 \\
\hline 2036 & 21 & 28994522.1 & 0.19865575 & 5759928.46 & 2061 & 46 & 58721624.24 & 0.0290073 & 1703355.54 \\
\hline 2037 & 22 & 30184472.68 & 0.18394051 & 5552147.21 & 2062 & 47 & 76623022.82 & 0.0268586 & 2057987.693 \\
\hline 2038 & 23 & 31374423.26 & 0.17031528 & \#NAME? & 2063 & 48 & 62493705.9 & 0.0248691 & 1554161.033 \\
\hline 2039 & 24 & 32564373.85 & 0.15769934 & 5135380.18 & 2064 & 49 & 63726058.66 & 0.0230269 & 1467415.291 \\
\hline 2040 & 25 & 33754324.43 & 0.1460179 & 4928735.74 & 2065 & 50 & 64958411.42 & 0.0213212 & 1384993.136 \\
\hline
\end{tabular}


Table 6 Discount rate of $10 \%$

\begin{tabular}{|c|c|c|c|c|c|c|c|c|c|}
\hline YEAR & $\mathbf{t}$ & $F=(O C+M / R C+R C)$ & $1(1+r)^{\wedge} t$ & $F+(1+r)^{\wedge} t$ & Year & $\mathbf{t}$ & $F=(O C+M / R C+R C)$ & $1(1+r)^{\wedge} t$ & $F(1+r)^{\wedge} t$ \\
\hline 2015 & 0 & 2865400 & 1 & & & & & & \\
\hline 2016 & 1 & 3348600 & 0.88495575 & 2963362.83 & 2041 & 26 & 34944275.02 & 0.0368877 & 1289015.265 \\
\hline 2017 & 2 & 3640000 & 0.69305016 & 2522702.59 & 2042 & 27 & 36062822.09 & 0.032644 & 1177235.342 \\
\hline 2018 & 3 & 3847060 & 0.61331873 & 2359473.94 & 2043 & 28 & 37313975.68 & 0.0288885 & 1077945.151 \\
\hline 2019 & 4 & 3026000 & 0.54275994 & 1642391.57 & 2044 & 29 & 38257237.63 & 0.0255651 & 978048.3033 \\
\hline 2020 & 5 & 12720050 & 0.48031853 & 6109675.68 & 2045 & 30 & 39660000.16 & 0.0226239 & 897265.4881 \\
\hline 2021 & 6 & 11651476 & 0.42506064 & 4952583.89 & 2046 & 31 & 40560029.59 & 0.0200212 & 812059.9119 \\
\hline 2022 & 7 & 13066616.29 & 0.37615986 & 4915136.58 & 2047 & 32 & 41999428.07 & 0.0177179 & 744140.1569 \\
\hline 2023 & 8 & 14673756.57 & 0.33288483 & 4884671.01 & 2048 & 33 & 43175672.26 & 0.0156795 & 676974.0633 \\
\hline 2024 & 9 & 18993341.3 & 0.29458835 & 5595217.04 & 2049 & 34 & 44370600.89 & 0.0138757 & 615672.5464 \\
\hline 2025 & 10 & 10389272.06 & 0.26069765 & 2708458.85 & 2050 & 35 & 45556721.14 & 0.0122794 & 559407.7691 \\
\hline 2026 & 11 & 14517669.52 & 0.23070589 & 3349311.84 & 2051 & 36 & 46742841.39 & 0.0108667 & 507940.3334 \\
\hline 2027 & 12 & 16355050.88 & 0.2041645 & 14473496.4 & 2052 & 37 & 47921203.62 & 0.0096165 & 460836.4953 \\
\hline 2028 & 13 & 31882417.3 & 0.18067655 & 5760405.19 & 2053 & 38 & 49114265.25 & 0.0085102 & 417973.1224 \\
\hline 2029 & 14 & 22927432.07 & 0.15989075 & 3665884.38 & 2054 & 39 & 50300354.09 & 0.0075312 & 378820.3517 \\
\hline 2030 & 15 & 18972446.83 & 0.14149624 & 2684529.92 & 2055 & 40 & 51486442.93 & 0.0066647 & 343144.2341 \\
\hline 2031 & 16 & 20017461.6 & 0.12521791 & 2506544.76 & 2056 & 41 & 53765790.75 & 0.005898 & 317111.0759 \\
\hline 2032 & 17 & 21612476.37 & 0.11081231 & 2394928.48 & 2057 & 42 & 53962740.52 & 0.0052195 & 281657.2454 \\
\hline 2033 & 18 & 22851568.52 & 0.09806399 & 2240916.06 & 2058 & 43 & 55152461.45 & 0.004619 & 254749.5271 \\
\hline 2034 & 19 & 26925820.44 & 0.08678229 & 2336684.49 & 2059 & 44 & 56342182.38 & 0.0040876 & 230305.1811 \\
\hline 2035 & 20 & 27804571.51 & 0.07679849 & 2135349.14 & 2060 & 45 & 57531903.31 & 0.0036174 & 208113.5424 \\
\hline 2036 & 21 & 28994522.1 & 0.06796327 & 1970562.43 & 2061 & 46 & 58721624.24 & 0.0032012 & 187979.8142 \\
\hline 2037 & 22 & 30184472.68 & 0.06014448 & 1815429.52 & 2062 & 47 & 76623022.82 & 0.0028329 & 217067.0949 \\
\hline 2038 & 23 & 31374423.26 & 0.05322521 & 31374423.3 & 2063 & 48 & 62493705.9 & 0.002507 & 156672.4189 \\
\hline 2039 & 24 & 32564373.85 & 0.04710195 & 1533845.6 & 2064 & 49 & 63726058.66 & 0.0022186 & 141382.2487 \\
\hline 2040 & 25 & 33754324.43 & 0.04168314 & 1406986.37 & 2065 & 50 & 64958411.42 & 0.0019634 & 127536.5832 \\
\hline
\end{tabular}

Table 7 Discount rate of $12 \%$

\begin{tabular}{|c|c|c|c|c|c|c|c|c|c|}
\hline Year & $\mathbf{t}$ & $\mathrm{F}=(\mathrm{OC}+\mathrm{M} / \mathrm{RC}+\mathrm{RC}$ & $1(1+r)^{\wedge} t$ & $F(1+r)^{\wedge} t$ & Year & $\mathbf{T}$ & $F=(O C+M / R C+R C)$ & 1.12 & $F(1+r)^{\wedge} t$ \\
\hline 2015 & 0 & 2865400 & 1 & & & & & $1(1+r)^{\wedge} t$ & \\
\hline 2016 & 1 & 3348600 & 0.892857 & 2989821.4 & 2041 & 26 & 34944275.02 & 0.052521 & 1835301.608 \\
\hline 2017 & 2 & 3640000 & 0.797194 & 2901785.7 & 2042 & 27 & 36062822.09 & 0.046894 & 1691114.827 \\
\hline 2018 & 3 & 3847060 & 0.71178 & 2738261.3 & 2043 & 28 & 37313975.68 & 0.041869 & 1562308.837 \\
\hline
\end{tabular}


World Journal of Advanced Engineering Technology and Sciences, 2021, 04(01), 040-051

\begin{tabular}{|c|c|c|c|c|c|c|c|c|c|}
\hline 2019 & 4 & 3026000 & 0.635518 & 1923077.7 & 2044 & 29 & 38257237.63 & 0.037383 & 1430180.825 \\
\hline 2020 & 5 & 12720050 & 0.567427 & 7217698 & 2045 & 30 & 39660000.16 & 0.033378 & 1323768.466 \\
\hline 2021 & 6 & 11651476 & 0.506631 & 5903000.3 & 2046 & 31 & 40560029.59 & 0.029802 & 1208758.554 \\
\hline 2022 & 7 & 13066616.29 & 0.452349 & 5910673.6 & 2047 & 32 & 41999428.07 & 0.026609 & 1117549.197 \\
\hline 2023 & 8 & 14673756.57 & 0.403883 & 5926484.2 & 2048 & 33 & 43175672.26 & 0.023758 & 1025756.695 \\
\hline 2024 & 9 & 18993341.3 & 0.36061 & 6849189.3 & 2049 & 34 & 44370600.89 & 0.021212 & 941201.3456 \\
\hline 2025 & 10 & 10389272.06 & 0.321973 & 3345067.6 & 2050 & 35 & 45556721.14 & 0.01894 & 862822.9048 \\
\hline 2026 & 11 & 14517669.52 & 0.287476 & 4173483.1 & 2051 & 36 & 46742841.39 & 0.01691 & 790435.2369 \\
\hline 2027 & 12 & 16355050.88 & 0.256675 & 4197934.2 & 2052 & 37 & 47921203.62 & 0.015098 & 723537.2231 \\
\hline 2028 & 13 & 31882417.3 & 0.229174 & 7306627.2 & 2053 & 38 & 49114265.25 & 0.013481 & 662098.7835 \\
\hline 2029 & 14 & 22927432.07 & 0.20462 & 4691406.9 & 2054 & 39 & 50300354.09 & 0.012036 & 605435.8844 \\
\hline 2030 & 15 & 18972446.83 & 0.182696 & 3466195.1 & 2055 & 40 & 51486442.93 & 0.010747 & 553314.4113 \\
\hline 2031 & 16 & 20017461.6 & 0.163122 & 3265281.6 & 2056 & 41 & 53765790.75 & 0.009595 & 515901.877 \\
\hline 2032 & 17 & 21612476.37 & 0.145644 & 3147734.9 & 2057 & 42 & 53962740.52 & 0.008567 & 462314.0001 \\
\hline 2033 & 18 & 22851568.52 & 0.13004 & 2971608.6 & 2058 & 43 & 55152461.45 & 0.007649 & 421880.9593 \\
\hline 2034 & 19 & 26925820.44 & 0.116107 & 3126270.2 & 2059 & 44 & 56342182.38 & 0.00683 & 384804.9656 \\
\hline 2035 & 20 & 27804571.51 & 0.103667 & 2882410 & 2060 & 45 & 57531903.31 & 0.006098 & 350830.8064 \\
\hline 2036 & 21 & 28994522.1 & 0.09256 & 2683721.7 & 2061 & 46 & 58721624.24 & 0.005445 & 319719.4203 \\
\hline 2037 & 22 & 30184472.68 & 0.082643 & 2494520.6 & 2062 & 47 & 76623022.82 & 0.004861 & 372487.9395 \\
\hline 2038 & 23 & 31374423.26 & 0.073788 & 28012878 & 2063 & 48 & 62493705.9 & 0.00434 & 271250.902 \\
\hline 2039 & 24 & 32564373.85 & 0.065882 & 2145409.4 & 2064 & 49 & 63726058.66 & 0.003875 & 246964.1692 \\
\hline 2040 & 25 & 33754324.43 & 0.058823 & 1985541 & 2065 & 50 & 64958411.42 & 0.00346 & 224767.8866 \\
\hline \multicolumn{10}{|c|}{ Salvage Value $=145707728.80$, Initial Cost $=5719455950.50$ LCC $=25712933.78$} \\
\hline
\end{tabular}

Table 8 Discount rate of $13 \%$

\begin{tabular}{|c|c|c|c|c|c|c|c|c|c|}
\hline Year & $\mathbf{t}$ & $F=(O C+M / R C+R C)$ & $1(1+r)^{\wedge} t$ & $F(1+r)^{\wedge} t$ & Year & $\mathbf{t}$ & $\mathrm{F}=(\mathrm{OC}+\mathrm{M} / \mathrm{RC}+\mathrm{RC})$ & $1(1+r)^{\wedge} t$ & $F(1+r)^{\wedge} t$ \\
\hline 2015 & & 2865400 & 1 & & & & & & \\
\hline 2016 & & 3348600 & 0.88495575 & 2963362.83 & 2041 & 26 & 34944275.02 & 0.0416831 & 1456587.25 \\
\hline 2017 & & 3640000 & 0.78314668 & 2850653.93 & 2042 & 27 & 36062822.09 & 0.0368877 & 1330275.937 \\
\hline 2018 & & 3847060 & 0.69305016 & 2666205.56 & 2043 & 28 & 37313975.68 & 0.032644 & 1218078.02 \\
\hline 2019 & & 3026000 & 0.61331873 & 1855902.47 & 2044 & 29 & 38257237.63 & 0.0288885 & 1105194.583 \\
\hline 2020 & & 12720050 & 0.54275994 & 6903933.52 & 2045 & 30 & 39660000.16 & 0.0255651 & 1013910.002 \\
\hline 2021 & & 11651476 & 0.48031853 & 5596419.79 & 2046 & 31 & 40560029.59 & 0.0226239 & 917627.7004 \\
\hline 2022 & & 13066616.29 & 0.42506064 & 5554104.33 & 2047 & 32 & 41999428.07 & 0.0200212 & 840878.3773 \\
\hline 2023 & & 14673756.57 & 0.37615986 & 5519678.24 & 2048 & 33 & 43175672.26 & 0.0177179 & 764980.6915 \\
\hline 2024 & & 18993341.3 & 0.33288483 & 6322595.25 & 2049 & 34 & 44370600.89 & 0.0156795 & 695709.9774 \\
\hline 2025 & 10 & 10389272.06 & 0.29458835 & 3060558.5 & 2050 & 35 & 45556721.14 & 0.0138757 & 632130.7791 \\
\hline 2026 & 11 & 14517669.52 & 0.26069765 & 3784722.37 & 2051 & 36 & 46742841.39 & 0.0122794 & 573972.5768 \\
\hline 2027 & 12 & 16355050.88 & 0.23070589 & 3773206.53 & 2052 & 37 & 47921203.62 & 0.0108667 & 520745.2397 \\
\hline 2028 & 13 & 31882417.3 & 0.2041645 & 6509257.87 & 2053 & 38 & 49114265.25 & 0.0096165 & 472309.6283 \\
\hline 2029 & 14 & 22927432.07 & 0.18067655 & 4142449.35 & 2054 & 39 & 50300354.09 & 0.0085102 & 428066.9975 \\
\hline
\end{tabular}




\begin{tabular}{|c|c|c|c|c|c|c|c|}
\hline 2030 & 1518972446.83 & 0.15989075 & 3033518.81 & 2055 & 4051486442.93 & 0.0075312 & 387752.9845 \\
\hline 2031 & 1620017461.6 & 0.14149624 & 2832395.58 & 2056 & 4153765790.75 & 0.0066647 & 358335.5158 \\
\hline 2032 & 1721612476.37 & 0.12521791 & 2706269.18 & 2057 & 4253962740.52 & 0.005898 & 318272.6873 \\
\hline 2033 & 1822851568.52 & 0.11081231 & 2532235.15 & 2058 & 4355152461.45 & 0.0052195 & 287866.9656 \\
\hline 2034 & 1926925820.44 & 0.09806399 & 2640453.47 & 2059 & 4456342182.38 & 0.004619 & 260244.8547 \\
\hline 2035 & $\begin{array}{lll}20 & 27804571.51 \\
\end{array}$ & 0.08678229 & 2412944.52 & 2060 & \begin{tabular}{|l|l|l}
45 & 57531903.31 \\
\end{tabular} & 205608.51 & 235168.3029 \\
\hline 2036 & 2128994522.1 & 0.07679849 & 2226735.54 & 2061 & \begin{tabular}{|l|l}
46 & 58721624.24 \\
\end{tabular} & 0.0036174 & 212417.19 \\
\hline 2037 & 2230184472.68 & 0.06796327 & 2051435.36 & 2062 & \begin{tabular}{|l|l}
47 & 76623022.82 \\
\end{tabular} & 0.0032012 & 245285.8172 \\
\hline 2038 & 2331374423.26 & 0.87100643 & 27764976.3 & 2063 & $\begin{array}{ll}48 & 62493705.9 \\
\end{array}$ & 0.0028329 & 177039.8333 \\
\hline 2039 & 2432564373.85 & 0.05322521 & 1733245.53 & 2064 & 4963726058.66 & 0.002507 & 159761.941 \\
\hline 2040 & 2533754324.43 & 0.04710195 & 1589894.6 & 2065 & $50 \quad 64958411.42$ & 0.0022186 & 144116.3391 \\
\hline
\end{tabular}

\subsection{Sensitivity analysis on the impact of discount rate in percentages}

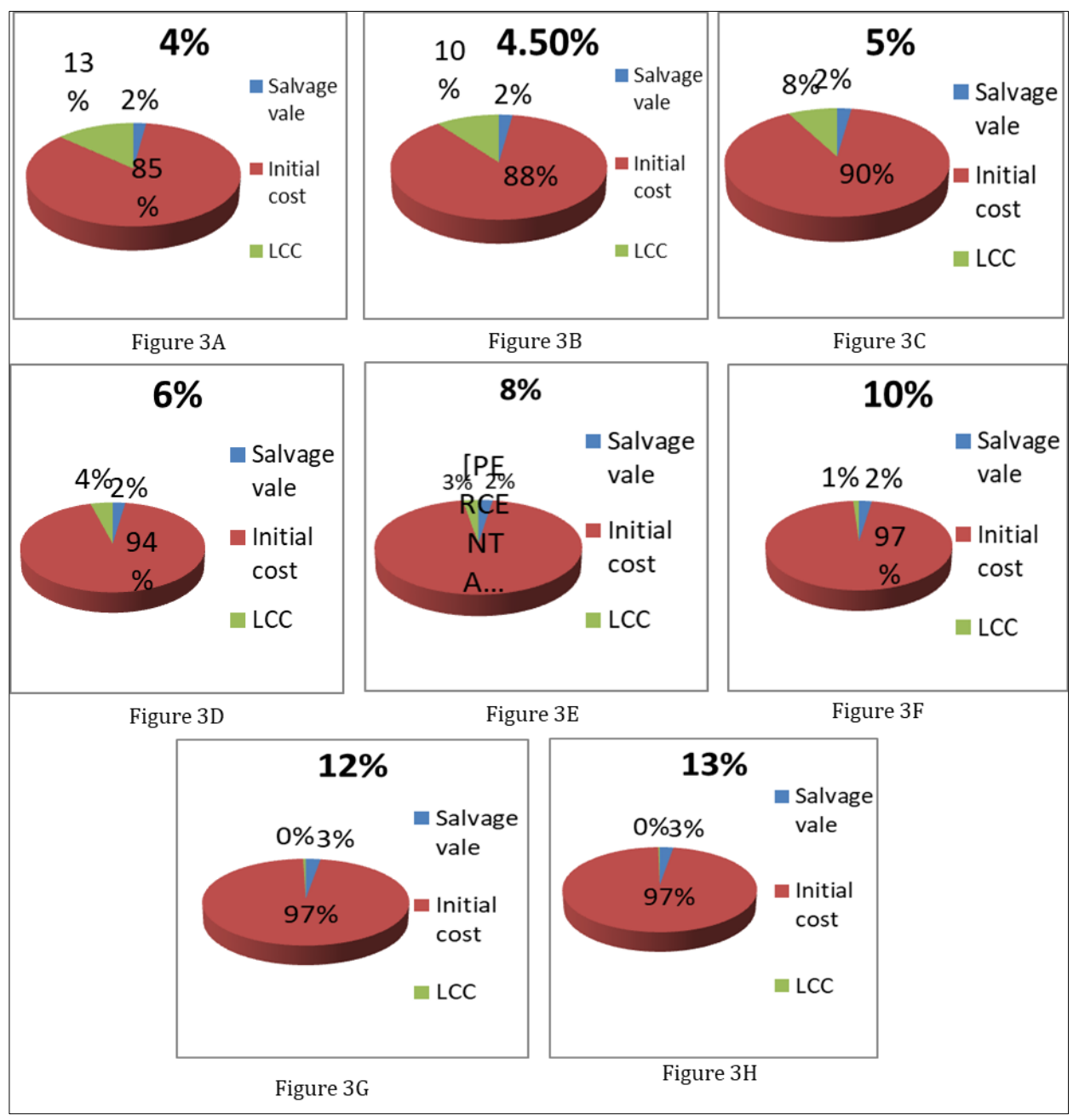

Figure 3A - 3H Percentage representation of discount rate

Percentage representation on the impact of different discount rate on the salvage value, initial cost and the life cycle cost of the project. The results from the pie chart which is obtain from table 3.1 to 3.1 .8 implies that the initial cost (construction cost) outweighed the life cycle cost and salvage value. Results from the discount rates literally shows that the life cycle cost decreases as the discount rate increases likewise the initial cost increases as the discount rate increases as show in figure 3.3. 


\section{Conclusion}

Life cycle costing enables the quantification of costs for relatively long period of time considering price changes. However, its implementation in real time is associated with some difficulties in obtaining the required cost data, particularly, if the analysis is conducted to develop life cycle cost in the nominal terms, in which future inflation and interest rates and the discount rates cannot be disregarded. The discount rates were applied to discount present value of the future cash flow. Thisresearch explains a theory in practice and demonstrates how the life cycle cost of a sustainable building was analyzed, estimated and discounted for a period of 51 years using different discount rates. Findings shows that the future costs associated with building operation, maintenance/repair and replacement at different discount rate generates different discount values.

\section{Compliance with ethical standards}

\section{Acknowledgments}

We wish to acknowledged the support of the Management of CCICC, Calabar for granting access to their facility to be used as a case study for this research, our colleagues who helped in the questionnaires, Prof. C.C Nnaji for proof reading the manuscript.

\section{Disclosure of conflict of interest}

There is no conflict of interest between the authors of this work and concerns exist anywhere pertaining to this work .

\section{References}

[1] Pernilla G, Henrikke B. The life cycle costing(LCC) approach: a conceptual discussion of its usefulness for environmental decision-making. 2004.

[2] Paldino and company, inc. king's country LCA guide. 2006.

[3] Flanagan R, Jewel C. Whole Life Appraisal for construction, Blackwell, Oxford. 2005.

[4] Langdon. Life cycle costing (LCC) as a contribution to sustainable construction: a common methodology literature review". Davis Langdon Management Consulting. NS 3454. 2007.

[5] Kelly J, Hunter J. Life Cycle Costing of Sustainable Design, RICS, London. 2009.

[6] Sophia A. Contribution of Life Cycle Cost analysis to design Sustainability in Construction. 2010.

[7] Jutta Schade. Life cycle cost calculation models for buildings. 2007.

[8] Ozbay K, Jawad D. The discount rate in life cycle cost analysis of transportation projects. 2006.

[9] Kirkham RJ, Alisa M, Silva A Pd, Grindley T, Brondsted J. Rethinking whole life cycle cost based design decisionmaking. In: Khosrowshahi F (Ed.), 20th Annual ARCOM Conference, 1-3 September 2004, Heriot Watt University. Association of Researchers in Construction Management. 2004; 1: 91-103.

[10] Luay N. Dwaikat, Kherun Nita Ali. Green buildings life cycle cost analysis and life cycle budget development Practical applications. 2004.

[11] Stephan A, Stephan L. Life cycle energy and cost analysis of embodied, operational and user-transport energy reduction measures for residential buildings. Applied Energy. 2016; 161: 445-464.

[12] Minja SJ. Developing a life cycle costing analytical method. 2016.

[13] Ugwu. Indicators and framework for assessment sustainable infrastructure. 2008.

[14] Herris F, McCaffer R. Modern construction management (5th ed.) 2005.

[15] World Bank, World Development Indicators, Inflation, Consumer Prices (Annual \%). 2019. 